\title{
TRANSFORMACIONES LÚDICAS. UN ESTUDIO PRELIMINAR SOBRE TIPOS DE JUEGO Y ESPACIOS LÚDICOS*
}

\section{Playful transformations. A preliminary study on type of PLAY and type of setting}

\author{
Graciela Stefanı $^{* *}$, Laura Andrés ${ }^{* * *}$ Y Estela Oanes***
}

\begin{abstract}
*Estudio realizado en el marco del proyecto El juego como herramienta para desarrollar habilidades sociales y creativas en niños dirgido por la Dra. María Cristina Richaud y coordinado por la Lic. Graciela Stefani. Este proyecto forma parte del convenio firmado entre CIIPME-CIIPCA y AGORA LUDUS.

**Licenciada en Psicología. Profesional del Sistema Nacional de Empleo Público (SINEP) del Consejo Nacional de Investigaciones Científicas y Técnicas (CONICET). E-Mail: gstefani@conicet.gov.ar

***Licenciada en Psicología. Profesional del Sistema Nacional de Empleo Público (SINEP) del Consejo Nacional de Investigaciones Científicas y Técnicas (CONICET). E-Mail: landres@conicet.gov.ar

****Licenciada en Psicología. Miembro de la Carrera del Personal de Apoyo a la Investigación del Consejo Nacional de Investigaciones Científicas y Técnicas (CONICET). E-Mail: estelaoanes@yahoo.com.ar

Las autoras agradecen a la Dra. María Cristina Richaud y a la Dra. María Luisa Silva la orientación y el asesoramiento recibidos y al Lic. Sergio Gentile, sus sugerencias para la realización de este estudio.

Centro Interdisciplinario de Investigaciones en Psicología Matemática y Experimental Dr. Horacio J.A. Rimoldi (CIIPME).

Tte. Gral. Perón 2158. (C1040AAH) Ciudad Autónoma de Buenos Aires. República Argentina.
\end{abstract}

\section{RESUMEN}

En la actualidad las modalidades lúdicas de los niños se han ido modificando. En las últimas décadas, en la Ciudad Autónoma de Buenos Aires se han producido cambios en los espacios lúdicos, que llevan a los niños a realizar cada vez más actividades en espacios privados o semipúblicos, haciendo variar su manera de jugar. El objetivo del estudio que se informa fue describir y analizar las transformaciones lúdicas, a partir de los juegos de los niños, los espacios y la percepción, y el recuerdo de personas pertenecientes a distintas generaciones. Participaron 516 sujetos de estrato social medio, agrupados en cuatro franjas de edad: niños, adolescentes, jóvenes y adultos y se les administró el Cuestionario Semiestructurado sobre Juegos (Stefani, Andrés \& Oanes, 2010). Los datos obtenidos fueron clasificados de acuerdo a los diferentes tipos de juegos y espacios lúdicos, a fin de describir semejanzas y diferencias entre las res- puestas dadas por los diferentes grupos de la muestra. Además se calcularon frecuencias y porcentajes de los juegos y de los espacios predominantes por edad. A fin de evaluar la asociación entre las variables tipo de juegos y tipo de espacios con las distintas franjas de edad, se aplicó la prueba de independencia de atributos chi-cuadrado. Los resultados permiten observar que los juegos se fueron transformando junto con los espacios donde se desarrollan, llevando a los niños a realizar sus prácticas cada vez más puertas adentro.

Palabras clave: Juego; Cultura lúdica; Transformaciones; Espacios lúdicos; Cambios generacionales.

\section{ABSTRACT}

Nowadays, children's play culture has changed. Over the last few decades, in Buenos Aires City, 
there have been many changes in play settings, which lead children to carry out activities in private or semi-public environments, modifying the way in which they play.

Children's play, understood as a space where children formerly trained their different skills, is being increasingly influenced by new technologies. At the same time, these new environments allow children to have new experiences. Children's play has been changing as time goes by; some games have been passed down, such as traditional games; some others have been modified, for example roleplaying, and others have disappeared or tend to do so. Digital options for children, which offer play and interactivity, are fully in line with the logic of consumption and they vary according to social economical level.

New generations incorporate these tools since their childhood, modifying childlike habits which held good for decades. We find that some decades ago, children used to play group games outdoors, on the streets around their neighborhood, at parks or at sport centers. Games such as elastics, hide and seek, skipping the rope, etc. were played on a daily basis while an adult was looking after the children. Nowadays, due to insecurity in the streets, children play indoors, in closed environments, or in private or institutional settings, and they play individually. The objective in conducting this study was to describe and analyze the changes in play habits which have been taking place, from information taken from research on children's play, play settings, and perception and recollection from subjects belonging to different generations.

In this study, 516 participants belonging to the middle class in Buenos Aires City (Argentina), who were grouped in four age ranges: young children, adolescents, young adults and adults, were given a semi-structured questionnaire (Stefani, Andrés \& Oanes, 2010). About play formats N and A which has been devised to build up knowledge about different types of play and play settings across generations. The constant comparison method was used to classify the obtained data into different types of play and play settings, in order to describe similarities and differences among the answers given by each study group. Furthermore, frequencies, averages, mean, median, and standard deviation prevailing in each age group were drawn from that. Statistically significant proof tests were per- formed: analysis of factor variance for the variable Type of play, and Chi-square Attribute Independence Test for the variable Type of setting. The results allow us to observe that play habits have been changing together with the places where they are developed, leading children to carry out their playful activities primarily indoors. Traditional games are shared by all generations, with slight variations, but without altering their essence, many of them consist of physical play like chase (chasing games), and hide and seek. Pretend / role-play has changed, as children use technology to play, increasingly incorporating electronic devices that grown-ups use for their work and social activities. In this way it is observed that play reflects and reproduces society.

We are in a moment of transition, changes in play habits and settings will make an impact on the way new generations build emotional and social skills. This leads us to think that this moment of transition, or experience crisis, comprises new possibilities, but at the same time, new risks such as individualism, isolation, negation of the other as a fellow human being, as the use of technology is basically an individual activity. A new virtual agora has been incorporated, with a new social order in which children are just one click away from socializing and opening the door to go out to play.

Key words: Play; Play culture; Transformations; Play settings; Cross-generational change.

\section{INTRODUCCIÓN}

Numerosos autores como Huizinga, Caillois, Vigotsky, desde diferentes disciplinas, han considerado que en todos los tiempos el juego es esencial para el desarrollo y la evolución de las personas. El contexto sociocultural e histórico y la época, de alguna manera, pueden caracterizarse por el juego.

A través del juego, el niño entrena y desarrolla sus recursos emocionales, cognitivos y creativos, en vista a la adultez. Sus experiencias lúdicas transitan por diferentes ámbitos: familiar, educativo y recreativo. 
Huizinga (2000) sostiene que el juego hace surgir la civilización, ya que no hay hombre sin juego, ni juego sin hombre. A través del juego el hombre se remonta a esa especial dimensión de alegría, a otro mundo donde se muestra la esencia de cada uno en forma libre y espontánea. Un valor esencial del juego y los juguetes es el de desarrollar y alimentar la imaginación.

En el Siglo XX y según la Teoría de la Recapitulación, Hall (ver Delval, 1994) dice que los niños reproducen a través del juego actividades que nuestros antepasados llevaron a cabo hace mucho tiempo, experimentando sumariamente la historia de la humanidad. Actividades tales como correr, jugar con arcos y flechas, trepar o esconderse serían entonces continuaciones o restos de actividades que fueron útiles o necesarias para la especie humana en otros tiempos.

Los juegos se han ido adaptando a las diferentes épocas. Los historiadores de juegos han identificado cuatro mecánicas universales presentes a lo largo de la historia en las diferentes culturas y que se corresponden con algunas actividades fundamentales de supervivencia y desarrollo del hombre. Estas mecánicas son la recolección, captura y caza, construcción e invención y habilidad y carreras. En los juegos existe una relación entre la estructura lúdica, las reglas de juego y la dinámica que reproducen este tipo de actividades (Parlett, 1999).

Pensadores clásicos como Platón y Aristóteles daban una gran importancia al aprender jugando, animando a los padres a dar a sus hijos juguetes que ayudaran a formar sus mentes para actividades futuras como adultos.

En este sentido, el fenómeno lúdico permite observar desde una óptica particular cómo ha ido evolucionando la humanidad y la niñez (Benjamín, 1989), ya que los valores culturales que se transmiten hacen que el juego se convierta en un espejo de la sociedad (Reboredo \& Espinosa, 1983).

Según Elkonin (1980), perteneciente a la escuela histórico-cultural de Vigotsky, lo fundamental en el juego es la naturaleza social de los papeles representados por el niño.
Caillois (1958) distribuye el juego en dos dimensiones contrapuestas según el modo de jugar: en una prevalece un principio común de diversión y turbulencia, improvisación y expansión en donde se manifiesta una fantasía incontrolada (paidia) y en la otra se hacen visibles la disciplina, las convenciones arbitrarias e imperativas que exigen esfuerzo, la paciencia y el ingenio (ludus). Ambos extremos se contraponen y se complementan. Para Schaines (1998), el juego es

"una actividad mágica, ata y desata energías, oculta y revela identidades, teje una trama misteriosa donde entes y fragmentos de entes, hilachas de universos contiguos y distantes, el pasado y el futuro, cosas muertas y aún no nacidas se entrelazan armoniosamente en un bello y terrible dibujo" (p. 14).

Para Winnicott (1999), el sujeto descubre la capacidad de transformar la realidad por medio de juegos creadores, empleo de símbolos y representaciones.

Los juegos de los niños han ido variando con el transcurrir del tiempo; algunos se han ido transmitiendo, otros modificando y otros han desaparecido o tienden a desaparecer. Pero hay algo que no varía, por ejemplo, los muñecos, autos, motos, cocinas, celulares, computadoras, porque estos siempre tratan de imitar nuestra forma de vida y los objetos que interesan a los adultos, haciendo que los niños de esta época varíen sus intereses, convirtiéndose en primordiales sujetos de consumo (Corea, 1999).

La disminución del tiempo de juego se produjo con la aparición del primer objeto electrónico en los años 30 con la llegada de la radio. Los chicos comienzan a no salir a la vereda para escuchar el radioteatro. Más tarde la llegada de la televisión arrasa con el tiempo de juego (Museo de la Ciudad, 2011) y ahora lo hace la tecnología.

Los juguetes son inseparables de la época y del contexto en el que fueron creados, constituyéndose en un elemento más de identidad. Khanna (2010) plantea que el jugar integra a la sociedad. A través del ju- 
guete, los pequeños aprenden la cultura y sus roles. La evolución del juguete está relacionada también con las transformaciones en la industria y los avances tecnológicos.

En nuestro país, los juguetes antiguamente no eran abundantes, muchos se construían de manera casera y a su vez la influencia de las comunidades inmigratorias, fenómeno que hoy se repite (Castro Solano, 2011, 2012), trajeron a la Argentina nuevas modalidades lúdicas y actualmente continúa siendo un lugar de consumo de juguetes y personajes importados.

Con el deseo de reproducir en el juego el mundo adulto es que el niño necesita compañeros de juego (Garaigordobil Landazábal, 1994). En el juego, el niño crea espacios, relaciones nuevas, vive situaciones diversas; es un laboratorio de comunicación social donde los niños reconstruyen el mundo de los adultos y sus complejas relaciones con el fin de dominarlo y comprenderlo (Ortega, 1987).

El juego además requiere de un espacio, es decir, un escenario físico, disponible para jugar y es tan importante como los juguetes. Según Silva (2004), los escenarios físicos pueden influir en el tipo de juego y en la calidad de las actividades lúdicas. Así, el juego motor y el juego libre son más frecuentes en escenarios externos; de esta manera la disponibilidad del espacio favorecería determinadas prácticas de juego.

El proceso de creciente urbanización que atraviesa la ciudad de Buenos Aires hace variar los espacios en los que los niños jugaban sin ningún temor. El juego en la actualidad se ha ido modificando, especialmente en las grandes ciudades, donde se concentran los grandes centros de consumo.

Varias décadas atrás, la ciudad de Buenos Aires contaba con mayor cantidad de viviendas con amplios patios, casas tipo chorizo ${ }^{1}$, conventillos con patios compartidos y

1 Vivienda típica de Buenos Aires y de algunas otras ciudades de Argentina que consiste en un patio lateral al que dan las habitaciones que están en hilera y conectadas entre sí. departamentos tipo casa. Esto propiciaba un lugar de encuentro para los niños preservando así un espacio de juego en común con vecinos y amigos de diferentes edades.

El espacio público (calles, parques, plazas) comienza a reducirse debido al aglomeramiento urbano de la ciudad y sus cambios en la infraestructura edilicia (Novick, Collado \& Favelukes, 2013).

Debido a la percepción de inseguridad, la mayoría de los niños sólo pueden jugar en la plaza si sus padres los acompañan y esto propicia que gran parte de su tiempo de ocio transcurra en el interior de los hogares y no en los espacios públicos, como se hacía antiguamente. Actualmente un rol protagónico en estas nuevas formas de vida pública lo desempeñan los malls o shoppings (Carli, 2006).

Con la industrialización que se produjo en el Siglo XIX, el juego sufrió fuertes modificaciones y a comienzos del Siglo XX los espacios de juego preferidos por los niños eran al aire libre, la calle, la vereda, donde predominaban los juegos tradicionales (Buenos Aires Toy Museum, 2012).

Actualmente los niños pasan la mayor parte de su tiempo en el colegio. A pesar de todos estos cambios, ellos tienen la misma necesidad de jugar que en diferentes épocas. La escuela como entorno lúdico, facilita cierto tipo de juegos a partir de los materiales y tiempos puestos a disposición de ellos, y dificulta otros, especialmente ciertos juegos motores que podrían ser peligrosos (Sarlé, 2006).

Según Calmels (2002), hay un aumento de los grupos institucionalizados por edad y clase social: por ejemplo, clubes, colonias, escuelitas, etc., lo que provoca una disminución de grupos heterogéneos y por lo tanto una reducción de los grupos espontáneos, caracterizados por albergar edades diferentes. La escuela es una generadora excepcional de grupos, es ahí donde se debe trabajar y recrear el valor del juego frente a la pérdida del espacio lúdico.

El tiempo libre de los niños transcurre cada vez más, puertas adentro, en espacios privados y semipúblicos y como consecuencia, sus actividades de ocio se van privati- 
zando e institucionalizando. Merced al ingreso de las diferentes tecnologías, las formas y los modos de relacionarse con los otros se van transformando. Así el amigo real se va transformando en el amigo virtual.

Los juegos de los niños estuvieron determinados por los cambios sociales y urbanísticos experimentados por las grandes ciudades. Antes era corriente ver a los niños jugar en plazas y calles al elástico, la escondida o la rayuela, cuyo objetivo era demostrar las habilidades para el salto, el equilibrio y demás destrezas físicas. Hoy, el ingreso de las nuevas tecnologías, la televisión, el aumento del tránsito, la inseguridad y la sobrecarga de tareas extraescolares impiden que los niños puedan seguir jugando en plena calle. Según Ofele (2008), los espacios disponibles en la Ciudad Autónoma de Buenos Aires son diversos, desde espacios abiertos, plazas con juegos, hasta juegotecas destinadas a la población infantil. Sin embargo, los espacios externos en nuestra sociedad actual se encuentran cada vez más restringidos, las plazas cuentan con menos espacios verdes y muchas veces se encuentran cercadas. Mientras que el juego más importante para un niño es explorar el mundo, trepar, ensuciarse, meterse, incitando a diferentes desafíos solo abriendo la puerta para ir a jugar, el ágora, lugar de encuentro, se ha ido transformando y con ello, los juegos y la socialización.

En la actualidad y con el surgimiento de las nuevas tecnologías, la llamada Generación $Z$ o Generación Web (niños nacidos entre 1996 y 2010) prefiere los juegos electrónicos y los espacios virtuales, ya que han crecido con las tecnologías que sus padres utilizaban para trabajar (Mascó, 2012). Si se decía que la Generación $X$ (nacidos entre 1964 y 1980) y la $Y$ (nacidos entre 1981 y 1995) fueron criadas por la generación Baby Boomers (nacidos entre 1945 y 1963) y la televisión, la Generación $Z$ está siendo criada por internet y los diferentes soportes electrónicos. Para los $Z$ o Nativos digitales, la vida transita a través de diferentes tipos de pantallas. El clásico abrir la puerta para ir a jugar de sus padres y abuelos, se va trans- formando en el ingreso al espacio virtual para desarrollar sus actividades lúdicas.

De acuerdo con el marco teórico reseñado, el estudio que se informa se propuso describir y analizar las transformaciones lúdicas a partir de los juegos de los niños, los espacios, la percepción y el recuerdo de sujetos pertenecientes a distintas generaciones.

\section{Metodología \\ MUESTRA Y PROCEDIMIENTO}

En este estudio participaron 516 sujetos (203 varones y 313 mujeres), residentes de la Ciudad Autónoma de Buenos Aires y pertenecientes a un estrato social medio. La selección de la muestra fue no aleatoria, intencional y dividida en grupos según rangos de edad: 206 niños de 7 a 12 años, 103 adolescentes de 13 a 18 años, 82 jóvenes de 19 a 29 años y 125 adultos mayores de 30 años (ver Tabla 1).

Los niños eran alumnos de dos escuelas privadas religiosas y los adolescentes, de dos escuelas públicas. Los jóvenes eran alumnos de dos universidades, una privada y otra pública. La muestra de adultos se conformó con padres y abuelos de los niños y adolescentes participantes del estudio.

Se realizó una charla informativa en la que se comunicó en qué consistía el cuestionario y el objetivo del estudio. La información se recabó durante los años 2011 y 2012. En todos los casos se garantizó la confidencialidad de los datos.

Luego de obtener el consentimiento escrito de los padres, el cuestionario fue administrado a los niños individualmente y en horario de clase. A los adolescentes y jóvenes se les administró el instrumento en forma grupal en las instituciones, y a los adultos se les envió el mismo para que lo completaran.

\section{INSTRUMENTO}

Se utilizó el Cuestionario Semiestructurado sobre Juegos (Stefani, Andrés \& Oanes, 2010) en sus dos versiones, Niños $(N)$ y 
Adolescentes, Jóvenes y Adultos $(A)$, diseñado para recabar información sobre los distintos tipos de juegos y juguetes utilizados a través de las distintas generaciones, los espacios lúdicos, materiales o elementos de juego, juegos individuales y grupales y los característicos del lugar de residencia, a efectos de explorar las preferencias generacionales.

El cuestionario está compuesto por 9 preguntas en la Versión $N$ y 11 en la Versión A, algunas de ellas son de tipo abierta y otras, de elección múltiple. Es de fácil aplicación, tanto individual como colectivamente y adecuado para todo ámbito, edad y formación. Las preguntas fueron revisadas por psicólogos especialistas a fin de estudiar la validez del contenido.

Para realizar este estudio se escogieron dos preguntas relativas a las categorías espacio y tipos de juegos y se preguntaba:

1.- a los niños “¿A qué jugás?” y al resto de los sujetos "¿A qué jugabas cuando eras chico?"

2.- a los niños “¿Dónde jugás?” y al resto de los sujetos "¿Dónde jugabas cuando eras chico?"

\section{ANÁLISIS DE DATOS}

Se seleccionaron las respuestas dadas por niños, adolescentes, jóvenes y adultos, con las cuales se indaga sobre los tipos de juego y los espacios lúdicos. Posteriormente se hizo el análisis de contenido para obtener una categorización de juegos. El mismo fue realizado independientemente por dos jueces expertos en la temática lúdica. Se analizó la confiabilidad entre jueces con la prueba Kappa de Cohen, obteniendo un índice de .95 , a fin de determinar la estabilidad de la clasificación.

Las respuestas se clasificaron según las categorías obtenidas y contabilizando la cantidad de menciones de juegos a fin de evaluar la intensidad de cada categoría. Se calcularon frecuencias y porcentajes de las respuestas dadas. Dichas categorías son las siguientes:
1.- Juego simbólico: En esta categoría se incluyeron juegos tales como: jugar a la mamá y al papá, a la secretaria, a la doctora, las representaciones de roles sociales o personajes, disfrazarse, etc. Estos juegos se caracterizan por utilizar un abundante simbolismo formado a partir de la imitación. E1 niño reproduce escenas de la vida real modificándolas de acuerdo a sus necesidades. A través de este tipo de juego, el niño internaliza los roles sociales, canaliza los conflictos y las angustias transformando lo real, por asimilación a las necesidades del yo (Piaget, 1961). Este tipo de juego consiste en hacer el como si de la realidad teniendo conciencia de esa ficción (Garaigordobil Landazábal, 1994).

2.- Juegos motores: En esta categoría se incluyeron juegos tales como jugar a las manchas, escondidas, juegos con pelota, rayuela, soga, payana, bolitas, etc. Son aquellos en los que el movimiento intenso y la puesta en juego de las capacidades motoras constituyen la característica más manifiesta e implican algún grado de compromiso corporal (Pavía, 1994) tanto global, como de motricidad fina y habilidad manual.

3.- Juegos de mesa: Esta categoría incluye juegos de ingenio, tales como ajedrez, skrabel, dominó, Ta Te Ti, cartas, etc. y utilizan como herramienta central un tablero $\mathrm{y} / \mathrm{o}$ fichas. En algunos se permite simbolizar el avance de los jugadores usando símbolos físicos. Algunos pueden implicar el uso de dados o naipes.

4.- Juegos electrónicos: Se considera juego electrónico a todo juego digital o interactivo con independencia de su soporte. En esta categoría se incluyeron juegos tales como Playstation, Wii, X-Box, PC, teléfonos celulares, etc. Estos juegos pueden emular reglas o características de los otros tipos de juego en un espacio virtual.

5.- Otros juegos: Esta categoría se constituye como residual y en ella se incluyeron rompecabezas, dibujar y pintar, armar pulseras, jugar con masa, ladrillitos, etc. y quedan incluidos todos los juegos de construcción, de habilidad y de creación donde se reconstruye el mundo con diversos elemen- 
tos, utilizando piezas de madera o de plástico como los Lego, o de montaje de pieza para reproducir barcos, aviones, dinosaurios, etc.

Siguiendo el mismo concepto de análisis de contenido y confiabilidad entre jueces $(K=.95)$ se categorizaron los siguientes espacios lúdicos: calle o vereda, casa, escuela, club, patio, plaza o parque, casa de familiares o amigos, otros espacios. En esta última categoría se incluyeron espacios con menores frecuencias de respuestas tales como: baldíos, playa, quinta, campo, etc.

A fin de evaluar la asociación entre las variables estudiadas y las diferentes franjas de edad, se aplicó la prueba de independencia de atributos chi-cuadrado.

\section{Resultados}

\section{TIPOS DE JUEGOS}

Se analizaron un total de 2.398 respuestas dadas por los 516 sujetos de la muestra. Los niños proporcionaron el $32.32 \%$ de las respuestas $(M=3.76)$, los adolescentes el $14.80 \%(M=3.45)$, los jóvenes el $23.44 \%$ $(M=6.85)$ y los adultos el $29.44 \%(M=5.65)$.

Como se observa en la Tabla 2 y en el Gráfico 1, los Juegos motores se mantienen con fuerte presencia a lo largo de todas las franjas de edad. Elaborando una jerarquía de preferencias lúdicas, de los adolescentes, jóvenes y adultos, seguidos de los Juegos motores, aparecen los Juegos simbólicos. En los niños, este lugar lo ocupan los Juegos electrónicos.

Las categorías de juegos menos elegidas son: por los niños, Otros juegos y los simbólicos; por los adolescentes, los Juegos de mesa y Otros juegos; por los jóvenes, los Electrónicos y los de Mesa y por los adultos, los Electrónicos y Otros juegos.

El Juego simbólico en los niños es el que más se diferencia de las otras franjas de edad, mostrando en ellos una gran disminución. Si bien los juegos motores mantienen una fuerte presencia en los cuatro grupos, son los adultos y los niños los que tienen un porcentaje de respuesta mayor en ellos.

Los niños mayoritariamente mencionan los Juegos electrónicos, diferenciándose ampliamente de los otros grupos de edad.

Los Juegos de mesa son mayormente mencionados por los adultos y los niños a diferencia del resto de las franjas y la categoría Otros juegos, por los jóvenes.

Se aplicó la prueba de independencia de atributos chi-cuadrado. Se observó que en todos los casos existe asociación estadísticamente significativa entre las variables tipos de juego y franjas de edad (ver Tabla 2).

\section{ESPACIOS LÚDICOS}

Se analizaron un total de 1.471 respuestas dadas a la pregunta correspondiente al Espacio lúdico. Los niños proporcionaron el $43.64 \%$ de las respuestas $(M=3.12)$, los adolescentes el $16.32 \%(M=2.33)$, los jóvenes el $16.11 \%(M=2.89)$ y los adultos el $23.93 \%(M=2.82)$.

Elaborando una jerarquía de preferencias por espacios lúdicos, se observa que los niños eligen la casa y la escuela como espacios para jugar. Los adolescentes mencionan mayormente la casa, seguidos por la casa de familiares o amigos como espacios lúdicos de su niñez. Los jóvenes, al igual que los niños, también preferían la casa y la escuela. En cuanto a los adultos, mencionan como espacio lúdico la calle / vereda en primer lugar seguido por la casa, marcando una significativa diferencia con el resto de las franjas.

Los espacios menos elegidos por los niños fueron: otros espacios, calle / vereda y casa de familiares o amigos; por los adolescentes: otros espacios y club; por los jóvenes: club, patio y otros espacios y en el caso de los adultos, otros espacios y patio.

El espacio calle / vereda fue el más elegido por los adultos de la muestra, diferenciándose ampliamente de los otros grupos de edad. La casa resultó ser altamente elegida como espacio lúdico por todas las franjas de edad, especialmente por los adolescentes. 
Fueron los niños y los jóvenes quienes más eligieron la escuela como espacio lúdico.

El club fue referido mayormente por los niños, al igual que el patio y la plaza o parque. Por último, las casas de familiares o amigos, fueron los lugares de juego más mencionados por los jóvenes y los adolescentes.

Para estudiar la asociación entre las variables tipos de espacios lúdicos y franjas de edad, se aplicó la prueba de independencia de atributos chi-cuadrado. Se observó que en todos los casos existe asociación estadísticamente significativa (ver Tabla 3 y Gráfico 2).

\section{DIscusıón}

Los resultados obtenidos revisten particular relevancia en tanto muestran las semejanzas y diferencias entre los tipos de juegos y los espacios lúdicos a través de distintas generaciones.

Para Dilthey (1947) desde una concepción historicista, una generación presupone un grupo de individuos nacidos en torno a una determinada fecha y perteneciente a una comunidad de educación y similares formas de relación.

El análisis y procesamiento de las respuestas muestran cómo algunos tipos de juego han sido conservados y transmitidos a través de las generaciones y, aun cuando el avance tecnológico ingresó en el espectro lúdico, parecería que no pudo desplazar a otros modos de jugar.

Se observa que los juegos motores han sido los más mencionados por todas las generaciones. Dentro de este tipo de juego se encontró que muchos de ellos son los denominados juegos tradicionales, como por ejemplo, escondida, manchas, rayuela, etc. Como plantea Lavega Burgués (citado en Ofele, 1999), resulta difícil disociar el juego tradicional del comportamiento humano.

Estos juegos son aquellos que desde mucho tiempo atrás son transmitidos de generación en generación, de abuelos a padres y de padres a hijos, y perduran a veces manteniendo su esencia, aunque con algunas modificaciones. Hoy es común ver a los chicos jugando diversos tipos de manchas, que toman diferentes nombres, o agregando algún tipo de variaciones como la sardinita, que es una variante de la escondida o el zapatito blanco, otra versión del pisa pizzuela.

A través de los juegos tradicionales se transmiten características, valores y formas de vida y tradiciones de diferentes lugares (Ofele, 1998). Cañeque (1993) afirma que hay ciertos tipos de juegos que han sobrevivido a la aparición masiva de los más sofisticados y tecnificados. Su valor resulta indiscutible porque estos juegos explican y ayudan a comprender la historia más profunda del hombre.

En el pasado se exploraba más con el cuerpo, se ejercitaba la destreza física, había menos juguetes y todo servía para jugar. Los deportes son considerados por los niños de esta muestra, como juegos.

En esta época en la que los adultos utilizan cada vez más los soportes digitales para distintas actividades laborales, sociales o recreativas, los niños incorporan cada vez más los juegos con soportes electrónicos, reproduciendo así el mundo adulto (Duek, 2010, 2012).

De esta manera, y coincidiendo con Reboredo y Espinosa (1983) se observa que en el juego se reproduce y se refleja la sociedad.

Como se destacó anteriormente, los juegos motores son los que predominan en todas las generaciones. Coincidiendo con Mascó (2012), en la muestra se observó que sólo los nativos digitales (niños nacidos entre 1994 y 2004) difieren del resto de las generaciones y prefieren los juegos electrónicos en lugar de los simbólicos. De acuerdo con Cabañes (2012), el mundo se transforma constantemente a través de las tecnologías, y mientras que antes los juegos colectivos tenían lugar en un espacio analógico, actualmente lo tienen cada vez más en un espacio digital.

Dentro de la categoría Otros juegos se incluyeron los juegos de construcción, por tener escasa presencia en la muestra estudiada. Mientras en generaciones anteriores cualquier material podría transformarse en juguete o juego, haciendo intervenir la imagi- 
nación, hoy el juego de construcción se ve notablemente disminuido. Este tipo de juego, junto con el simbólico, son los que actualmente tienen menor presencia en los niños. Los niños actuales poseen una herramienta nueva y poderosa para indagar, explorar, analizar, expresarse y jugar. El resultado es una nueva cultura que va desplazando a la de sus padres (Tapscott, 1998).

En relación a los espacios, los juegos se han ido desplazando de los lugares públicos, abiertos y de ocio a los cerrados e institucionales (Calmels, 2002). Esto se ve reflejado en el estudio realizado. El dormitorio hoy se ha convertido en un espacio de ocio y experimentación enriquecido por la presencia de las nuevas tecnologías.

Hay una modificación de la concepción espacio-temporal, una nueva cultura que genera nuevas experiencias con nuevos espacios. Se ha incorporado una nueva ágora virtual con un nuevo ordenamiento social. En relación a los niños, los adultos suelen tener una representación diferente de los espacios urbanos (potreros, baldíos, etc.) y de los juegos que evocan. La calle como espacio de diversidad también es productora de experiencias diversas. La experiencia urbana, entre otras cosas, promueve un contacto directo con las diferencias sociales, culturales y étnicas.

Coincidiendo con el Observatorio de la Deuda Social Argentina (2009), la gran mayoría de los niños en edad escolar juega en su propia casa y tiene como espacios alternativos la escuela, el patio y la plaza y en menor medida, casas de familiares o de amigos, calle / vereda y club. La casa de amigos y familiares son espacios de juego más frecuentes en el estrato medio y medio-alto.

Los espacios públicos cercanos a las viviendas, como vereda y calle fueron los escenarios de juego de los adultos actuales, este fenómeno también se observa en el estudio realizado. El lugar donde menos juegan los niños, es en espacios abiertos y públicos, como la calle / vereda, lugar de encuentro de grupos espontáneos y heterogéneos. Este cambio además, trae aparejado la pérdida del vecino como uno de los compañeros de juego de ayer. Es importante agregar que las nuevas tecnologías están desplazando y cambiando las formas tradicionales de transmisión y los agentes de socialización.

Se observa que los Juegos motores como trepar, saltar, correr, andar en patines y bicicleta que se entrenaban en espacios abiertos y públicos, presentan una importante disminución en los niños. La habilidad física actualmente se ubicaría en el terreno de la fantasía video lúdico, más que en el terreno de la realidad. Los juegos salieron de la calle y pasaron al interior de las casas por medio de los soportes tecnológicos. Estudios recientes (Martínez Reina \& Velez Cea, 2009) demuestran que los niños y niñas interactúan de forma diferente con estos soportes. Los juegos de acción, aventuras, carreras, estrategia, están más orientados al usuario masculino (Rodríguez San Julián, 2002), en tanto las niñas no se identifican tanto con este ofrecimiento y hacen mayor uso de las redes sociales.

En relación a esto, se observa que los videojuegos suponen un cambio de paradigma perceptivo que integra interactividad, hipermedia, hipertextualidad y conectividad $(\mathrm{Ca}-$ bañes, 2012). El juego electrónico permite experimentar empíricamente mediante la simulación de mundos ficticios, con lo cual la diferencia entre fantasía y realidad podría alterarse (Nabel \& González, 2009).

Una limitación encontrada en el estudio realizado es que la muestra de los niños fue evaluada en escuelas, por lo cual se podría suponer o pensar que el espacio Patio al que refieren los niños, se correspondería con el patio escolar, siendo este un espacio institucional y cerrado. La escuela cumple o cubre diferentes roles con la doble jornada, convirtiéndose en el lugar donde los niños pasan la mayor parte de su tiempo.

En relación al espacio virtual, el mismo no ha sido clasificado a priori como tal, sino que surge de las respuestas dadas por los niños a la pregunta relacionada con la variable Tipos de juegos. La web, como plaza pública de encuentro o intercambio, genera lazos emocionales de pertenencia a un determinado colectivo o grupo social, caracterizados por la 
inmediatez, rápida interacción y una comunicación impersonal (Area \& Posea, 2012).

Para los adultos y parte de los jóvenes de la generación Baby Boomers, $X$ e $Y$ (Mascó, 2012), la experiencia lúdica transcurría en espacios públicos o semipúblicos tales como plazas, calles y veredas. Los Nativos digitales la desarrollan en espacios cerrados o privados tales como la escuela, la habitación, colonias y el club, correspondiéndose estos espacios con los niveles de ingresos de la familia. En este contexto, se ha incorporado un nuevo espacio de juego al que hacen mención los chicos: el shopping y al que concurren acompañados por adultos. Además, merced al ingreso de las nuevas tecnologías, se ha incorporado un nuevo espacio lúdico y social: el ágora virtual. Se destaca que la computadora se ha convertido en un objeto más de juego para los chicos.

Es el adulto quien proporciona o no, las diferentes alternativas lúdicas a los niños y el acceso a determinados espacios, favoreciendo u obstaculizando ciertas prácticas lúdicas. Se observa en el estudio realizado que el juego por excelencia que acerca a las generaciones es el juego tradicional.

Debido a los cambios en los patrones de interacción familiar y la suma de aparatos tecnológicos, se ha generado una cultura de habitación, antes vinculada a las mujeres. Este espacio se convierte en un espacio privado, íntimo, rico tecnológicamente y lugar de la experimentación personal, observándose la web como ecosistema artificial para la experiencia humana.

El niño actual mira al mundo a través de una pantalla, con más amigos virtuales que reales, con pérdidas de grupos espontáneos y una nueva manera de jugar que incluye otros mediadores tecnológicos.

Dado que esta muestra es reflejo de un estrato social medio, sería interesante reproducir la investigación en otros contextos y estratos sociales.

Se concluye que los juegos han viajado por el mundo, adaptándose, mezclándose con las distintas culturas, respondiendo y haciendo converger en su formalidad de juego el momento histórico determinado en el cual eran conocidos.

TABLA 1

ESTADÍSTICOS DESCRIPTIVOS DE LA MUESTRA

\begin{tabular}{|l|cccc|}
\hline & Niños & Adolescentes & Jóvenes & Adultos \\
& & & & 125 \\
$\%$ & 206 & 103 & 82 & 24.2 \\
\hline Rango de edad & 39.9 & 20 & 15.9 & \\
$M$ & $7-12$ & $13-18$ & $19-29$ & 30 ó más \\
$D E$ & 9.81 & 15.45 & 20.77 & 44.37 \\
& 1.50 & 1.49 & 2.44 & 9.86 \\
\hline
\end{tabular}


TABLA 2

Tipos de Juego Por Franjas de EdAd. Distribución de fRECUENCIAS Y PORCENTAJes dE RESPUESTAS

\begin{tabular}{|c|c|c|c|c|c|c|c|}
\hline Franjas de edac & o de juego & $\begin{array}{c}\text { Juego } \\
\text { simbólico }\end{array}$ & $\begin{array}{l}\text { Juegos } \\
\text { motores }\end{array}$ & $\begin{array}{l}\text { Juegos } \\
\text { electrónicos }\end{array}$ & $\begin{array}{l}\text { Juegos } \\
\text { de mesa }\end{array}$ & $\begin{array}{c}\text { Otros } \\
\text { juegos }\end{array}$ & Total \\
\hline $\begin{array}{l}\text { Niños } \\
n=206\end{array}$ & $\begin{array}{l}n \\
\%\end{array}$ & $\begin{array}{c}59 \\
7.61\end{array}$ & $\begin{array}{c}368 \\
47.48\end{array}$ & $\begin{array}{c}234 \\
30.19\end{array}$ & $\begin{array}{c}80 \\
10.32\end{array}$ & $\begin{array}{c}34 \\
4.39\end{array}$ & $\begin{array}{l}775 \\
100\end{array}$ \\
\hline $\begin{array}{l}\text { Adolescentes } \\
n=103\end{array}$ & $\begin{array}{l}n \\
\%\end{array}$ & $\begin{array}{c}146 \\
41.13\end{array}$ & $\begin{array}{c}157 \\
44.23\end{array}$ & $\begin{array}{c}19 \\
5.35\end{array}$ & $\begin{array}{c}15 \\
4.23\end{array}$ & $\begin{array}{c}18 \\
5.07\end{array}$ & $\begin{array}{l}355 \\
100\end{array}$ \\
\hline $\begin{array}{l}\text { Jóvenes } \\
n=82\end{array}$ & $\begin{array}{l}n \\
\%\end{array}$ & $\begin{array}{c}230 \\
40.93\end{array}$ & $\begin{array}{c}255 \\
45.37\end{array}$ & $\begin{array}{c}10 \\
1.78\end{array}$ & $\begin{array}{c}23 \\
4.09\end{array}$ & $\begin{array}{c}44 \\
7.83\end{array}$ & $\begin{array}{l}562 \\
100\end{array}$ \\
\hline $\begin{array}{l}\text { Adultos } \\
n=125\end{array}$ & $\begin{array}{l}n \\
\%\end{array}$ & $\begin{array}{c}181 \\
25.64\end{array}$ & $\begin{array}{c}411 \\
58.22\end{array}$ & $\begin{array}{l}4 \\
.57\end{array}$ & $\begin{array}{c}77 \\
10.91\end{array}$ & $\begin{array}{c}33 \\
4.67\end{array}$ & $\begin{array}{l}706 \\
100\end{array}$ \\
\hline $\begin{array}{l}\text { Total } \\
N=516\end{array}$ & $\begin{array}{l}n \\
\%\end{array}$ & $\begin{array}{c}616 \\
25.69\end{array}$ & $\begin{array}{l}1.191 \\
49.67\end{array}$ & $\begin{array}{c}267 \\
11.13\end{array}$ & $\begin{array}{c}195 \\
8.13\end{array}$ & $\begin{array}{l}129 \\
5.38\end{array}$ & $\begin{array}{c}2.398 \\
100\end{array}$ \\
\hline
\end{tabular}

Nota:

$\chi^{2}(12)=216.11, p<.001$ 
TABLA 3

TIPOS DE ESPACIOS LÚDICOS POR FRANJAS DE EDAD: DISTRIBUCIÓN DE FRECUENCIAS Y PORCENTAJES

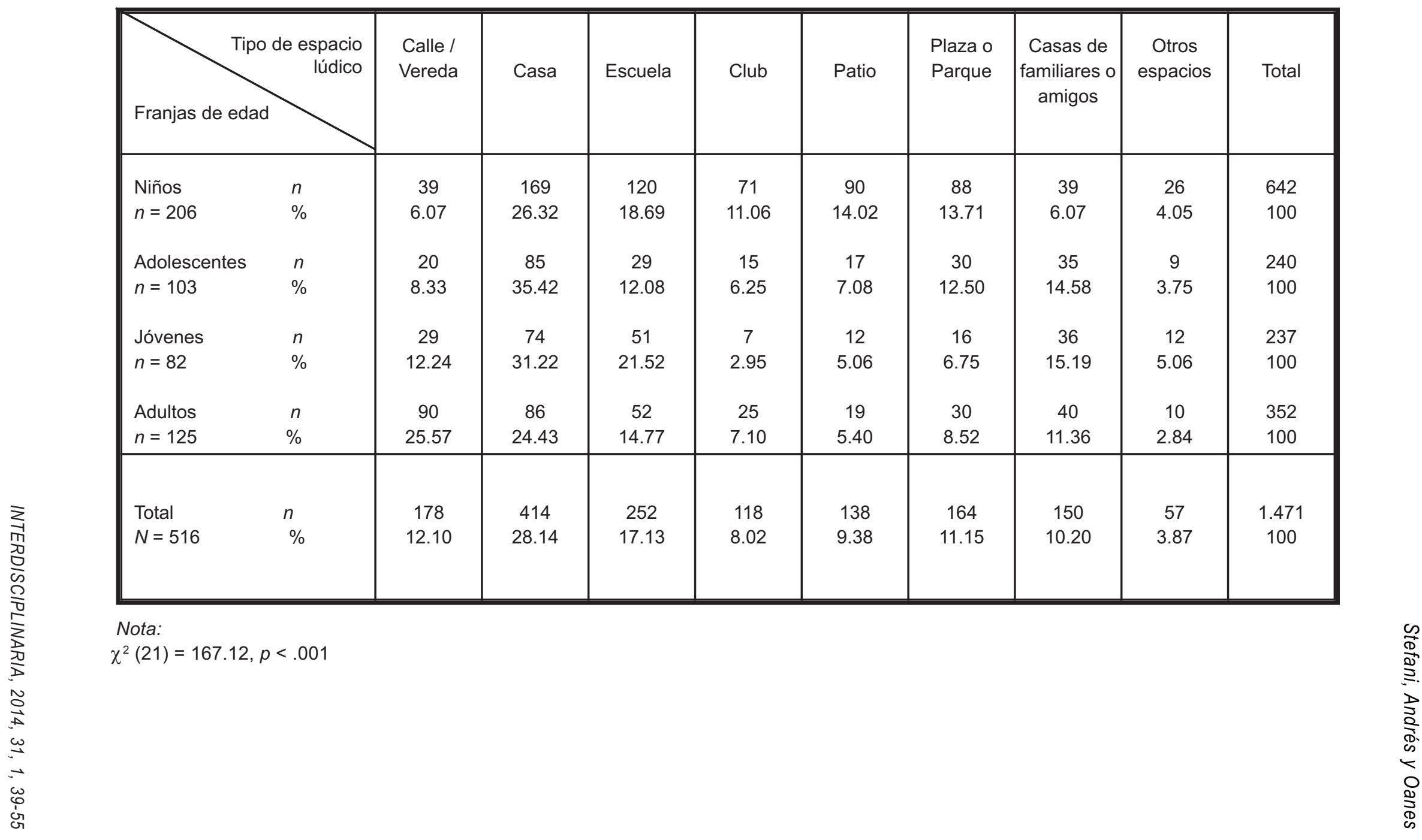




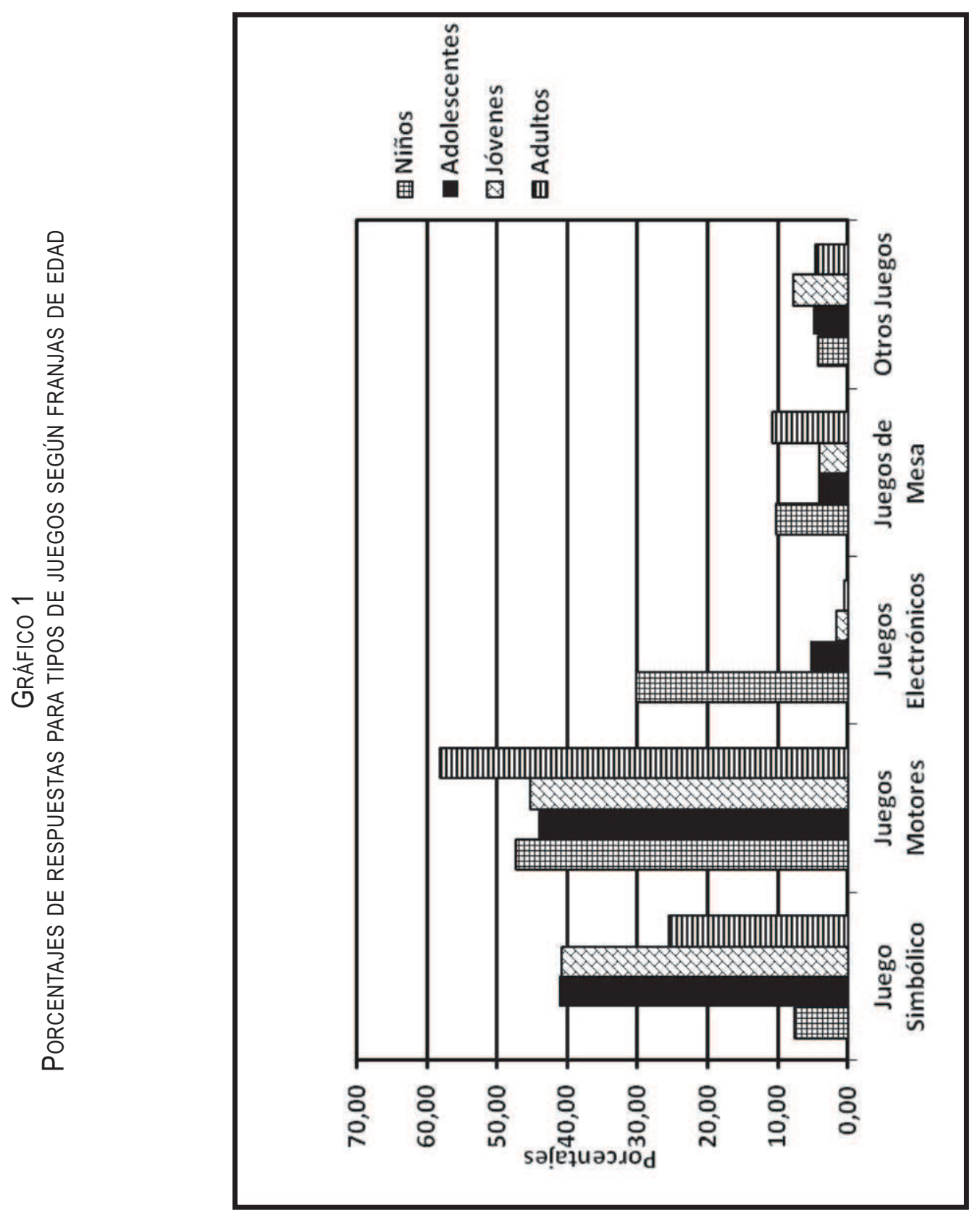




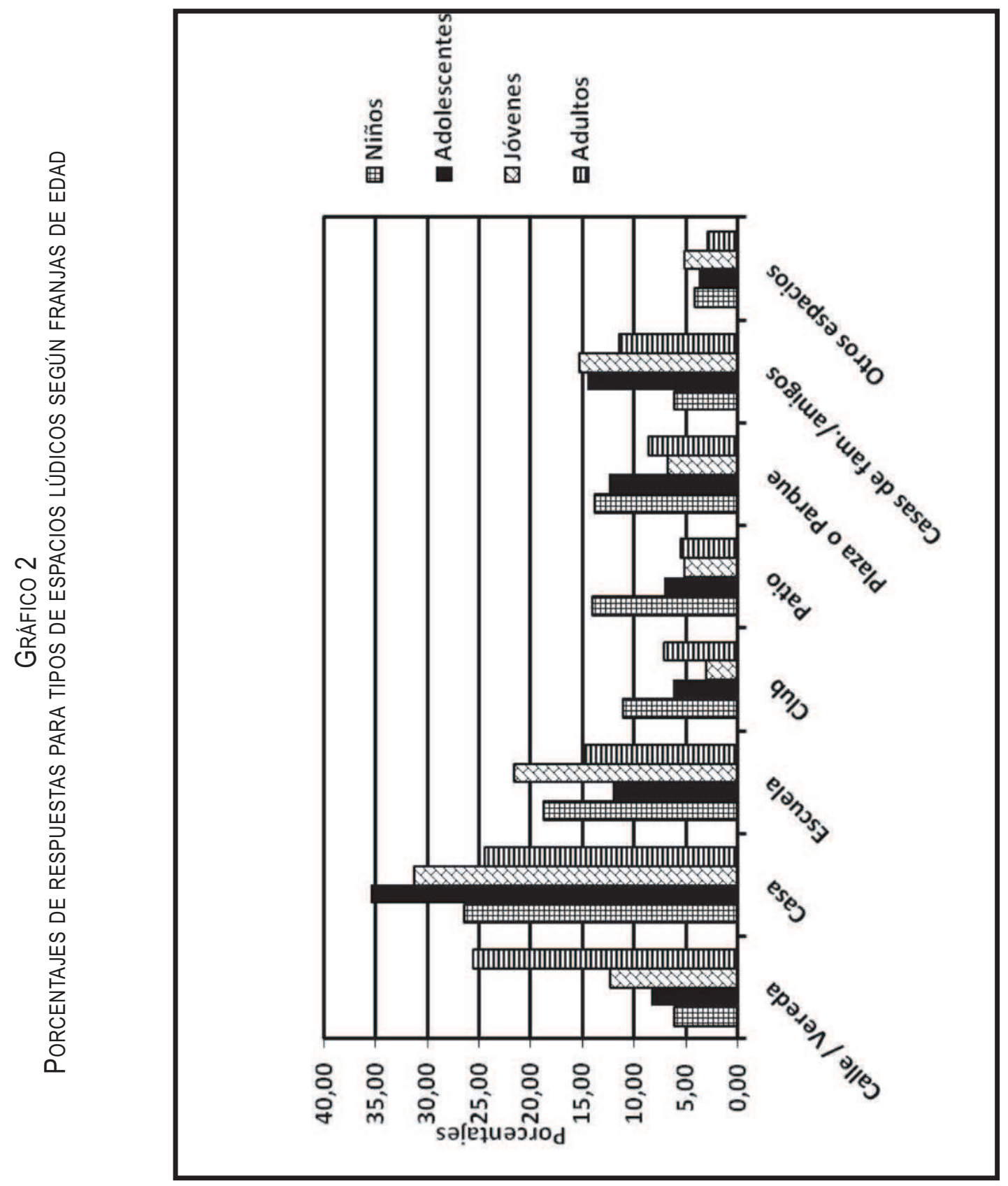




\section{REFERENCIAS BIBLIOGRÁFICAS}

Area, M. \& Posea, T. (2012). De lo sólido a lo líquido: Las nuevas alfabetizaciones ante los cambios culturales de la web 2.0 [From solid to liquid: New literacies to the cultural changes of web 2.0]. Comunicar, 19(38), 13-20. Recuperado el 10 de junio de 2013 de http:// www.revista comunicar.com/ index.php?con tenido $=$ detalles $\&$ numero $=38 \&$ articulo $=38$ 2012-03. http://dx.doi.org/10.39 16/c38-201202-01

Benjamin, W. (1989). Escritos: La literatura infantil, los niños y los jóvenes [Writings: Children's literature, children and youth]. Buenos Aires: Nueva Visión.

Buenos Aires Toy Museum. (2012). La historia del juguete [Toy story]. Recuperado el $24 \mathrm{de}$ febrero de 2013 de http://www.the-ba-toymu seum.comel

Cabañes, E. (2012). Del juego simbólico al videojuego: La evolución de los espacios de producción simbólica [Symbolic play the game: The evolution of symbolic production spaces]. Revista de Estudios de Juventud, 98, Documento 5. Recuperado el 13 de junio de 2013 de http://euridicecabanes.es.t1/Del-juegosimb\%F3lico-al-videojuego.htm

Caillois, R. (1958). Los juegos y los hombres: La máscara y el vértigo [Play and men: The mask and the vertigo]. Buenos Aires: Fondo de Cultura Económica.

Calmels, D. (2002). Trastornos en el desarrollo infantil: Algunas reflexiones interdisciplinarias [Childhood developmental disorders: Some interdisciplinary reflections]. En J. Tallis \& J. C. Reboiras (Comps.), Periplo de un hombre: Desatención - hiperactividad - impulsividad (Cap. 3). Buenos Aires: Miño y Dávila.

Cañeque, H. (1993). Juego y vida. La conducta lúdica en el niño y el adulto [Play and life. Play behavior in children and adults]. Buenos Aires: El Ateneo.
Carli, S. (2006). La cuestión de la infancia: Entre la escuela, la calle y el shopping [The issue of childhood: Between the school, the street and the mall]. Buenos Aires: Paidós.

Corea, C. (1999). ¿Se acabó la infancia? [Is childhood over?]. Buenos Aires: Lumen.

Castro Solano, A. (2011). Estrategias de aculturación y adaptación psicológica y sociocultural de estudiantes extranjeros en la Argentina [Acculturation strategies and psychological and sociocultural adaptation of foreign students in Argentina]. Interdisciplinaria, 28(1), 115-130.

Castro Solano, A. (2012). La evaluación de las competencias culturales: Validación del ICC [Assessment of cultural competente: Validation of the inventory ICC]. Interdisciplinaria, 29(1), 109-132.

Delval, J. (1994). El desarrollo humano [Human development]. Madrid: Siglo Veintiuno.

Dilthey, W. (1947). Le monde de l'esprit (1875), t.l. Histoire de sciences humaines [The world of the spirit (1875). History of human sciences]. París: Aubier Montaign.

Duek, C. (2010, marzo). Infancia, medios de comunicación y juego: Un campo de trabajo, un objeto de estudio [Childhood, media and play: A work field and an object of study]. Hebrew University of Jerusalem, 3(1), 53-74.

Duek, C. (2012). El juego infantil contemporáneo: Medios de comunicación, nuevas prácticas y clasificaciones [Contemporary child's play: Communication media, new practices and classifications]. Revista Brasileña Ciencias Esporte, 34(3), 649-654.

Elkonin, D. (1980). Psicología del juego [Psychology game]. Madrid: Pablo del Río.

Garaigordobil Landazábal, M. (1994). Evaluación de una intervención psicoeducativa en sus efectos sobre la conducta prosocial y la creatividad [Evaluation of a psychoeducational intervention in its effects on prosocial behaviour and creativity]. Madrid: CIDE. 
Huizinga, J. (2000). Homo Ludens [Homo Ludens]. Madrid: Alianza. http://dx.doi.org/ 10.5117/9789089640031

Khanna, S. (2010, agosto). Cómo, por qué y para qué jugar: El juego dentro y fuera de la escuela [How, why, and what playing is for: Play in school and outside of school]. Trabajo presentado en el Seminario Internacional: $\mathrm{La}$ infancia, el juego y los juguetes. FLACSO: Buenos Aires, Argentina.

Martínez-Reina, M.C. \& Velez Cea, M. (2009). Estereotipos de género en el juego y en el ocio tecnológico interactivo [Gender stereotypes in the game and interactive entertainment technology]. Granada: Universidad de Granada.

Mascó, A. (2012). Entre generaciones. No te quedes afuera del futuro [Between generations. Don't be left out of future]. Buenos Aires: Temas.

Museo de la Ciudad. (2011). Exposición permanente de patrimonio: Los porteños vuelven a jugar y a divertirse con los juguetes de ayer [Permanent exhibition of patrimony: Buenos Aires citizens play and have fun again with yesterday's toys]. Buenos Aires: Biblioteca CABA.

Nabel, N. \& González, J. (2009). El juego hoy. Incidencia de las nuevas tecnologías. Repercusiones e interrogantes [Today's play. The influence of new technologies. Repercussions and inquiries]. Revista Topía. Recuperado el 5 de enero de 2013 de www.topia.com.ar/articu los/eljuegohoy

Novick, A., Collado, F. \& Favelukes, G. (2013). Urbanización AMBA [Urbanization AMBA]. Atlas Ambiental de Buenos Aires. Recuperado el 12 de febrero de 2013 de http://www.atlasd ebuenosaires.gov.ar

Observatorio de la Deuda Social Argentina. (2009). Argentina 2004-2008: Condiciones de vida de la niñez y adolescencia [Argentine 2004-2008: Living conditions in childhood and adolescence]. Barómetro de la Deuda So- cial de la Infancia, Departamento de Investigación Institucional. Buenos Aires: Pontificia Universidad Católica Argentina.

Ofele, M. (1999). Los juegos tradicionales y sus proyecciones pedagógicas [Traditional play and educational projections]. Lecturas: Educación Física y Deportes. Revista Digital, 4 (13), 1. Recuperado el 14 de mayo de 2013 de http://www.efdeportes.com/efd13/juegtra 1.htm

Ofele, M. (2008). Repensar la niñez en el siglo XXI. Los espacios del juego y el jugar en la sociedad actual [Rethinking childhood in the $X X I$ Century. Play settings and play in today's society]. Recuperado el 30 de abril de 2013 de http://A6N2\%20julio\%2008.pdf

Ortega, R. (1987). El juego: Un laboratorio de comunicación [Play: A communication laboratory]. Actas de las V Jornadas de Estudio sobre la Investigación en la Escuela. Sevilla, España: Servicio de Publicaciones de la Universidad de Sevilla.

Parlett, D. (1999). The Oxford history of board games. Oxford: Oxford University Press.

Pavía, V. (1994). Juegos que vienen de antes [Games from earlier times]. Buenos Aires: Humanitas, Cosmovisión.

Piaget, J. (1961). La formación del símbolo en el niño [The formation of the symbol in the child]. México: Fondo de Cultura Económica.

Reboredo, A. \& Espinosa, A. (1983). Jugar es un acto político: El juguete industrial, recurso de dominación [Playing is a political act: The toy industry, resource domination]. México: Centro de Estudios Económicos y Sociales del Tercer Mundo. Nueva Imagen.

Rodríguez San Julián, E. (2002). Jóvenes y videojuegos: Espacio, significación y conflictos [Young adults and videogames: Space, significance and conflict]. Madrid: Fundación de Ayuda contra la Drogadicción (FAD). Recuperado el 3 de enero de 2012 de http://www.f 
ad.es/sala_lectura/videojuegos.pdf sala_lectura/videojuegos.pdf

Sarlé, P. (2006). Enseñar el juego y jugar la enseñanza [Teaching the game and play teaching]. Buenos Aires: Paidós.

Schaines, G. (1998). Juegos inocentes, juegos terribles [Innocent games, terrible games]. Buenos Aires: Eudeba.

Silva, G. (2004). El juego como estrategia para alcanzar la equidad cualitativa en la educación inicial. Entornos lúdicos y oportunidades de juego en el CEI y la familia [The game as a strategy to reach qualitative equality in initial education. Recreational environments and play opportunities in the CEI and family]. Recuperado el 25 de abril de 2013 de http:// www.flacso.org.ar./ar/libros/peru/grade/edu ca/doc4.pdf
Stefani, G., Andrés, L. \& Oanes, E. (2010). Cuestionario Semiestructurado sobre Juegos [Semistructured questionnaire about games]. Manuscrito inédito.

Tapscott, D. (1998). Creciendo en un entorno digital. La generación internet [Growing in a digital environment. The internet generation]. Bogotá: Mac Graw Hill.

Vigotsky, L.S. (1982). El papel del juego en el desarrollo. El desarrollo de los procesos superiores [The role of play in children's development. Development of higher processes]. Barcelona: Crítica.

Winnicott, D. (1999). Realidad y juego [Reality and play]. Buenos Aires: Gedisa-Psicoteca Mayor.

Centro Interdisciplinario de Investigaciones en Psicología Matemática y Experimental Dr. Horacio J.A. Rimoldi (CIIPME)

Consejo Nacional de Investigaciones Científicas y Técnicas (CONICET) Ciudad Autónoma de Buenos Aires República Argentina

Fecha de recepción: 27 de mayo de 2013 Fecha de aceptación: 21 de enero de 2014 
